Methods Among 1662 AP patients admitted between August 2010 and August 2017, 88 eligible subjects suffering from moderately severe and severe AP (SAP) were selected. Meanwhile, a well-balanced cohort of mild AP (MAP) patients was generated by propensity score matching at a ratio of 1:2. NAFPD was assessed by attenuation of the pancreas via unenhanced computed tomography (CT). In the end, 264 patients were analysed.

Results In all, 176 patients had MAP, 48 patients had moderate SAP, and 40 patients had SAP. Among them, the more severe the $\mathrm{AP}$, the lower the mean pancreatic attenuation and the pancreas attenuation/spleen attenuation $(\mathrm{P} / \mathrm{S})$ ratio (both $\mathrm{p}<0.001$ ) (figure 1). Pancreas attenuation decreased with increases in the rate of intensive care unit (ICU) transfer, the severity of AP, the systemic complications and the prognostic factors of AP (APACHE-II score $\geq 8 ; \mathrm{p}<0.001)$. A decreased $\mathrm{P} / \mathrm{S}$ ratio was positively correlated with the increased mortality of patients with AP (HR, 0.000; 95\% CI, 0.000-0.008, $\mathrm{p}<0.001$ ) according to Cox proportional hazard models adjusted for creatinine, calcium and albumin levels (table 1). Pancreas attenuation and the $\mathrm{P} / \mathrm{S}$ ratio exhibited high areas under the receiver operating characteristic (ROC) curves for the prediction of AP severity (pancreas attenuation: 0.932, 95\% CI, 0.893-0.972; P/S ratio: 0.891, 95\% CI, 0.846-0.936).

\begin{tabular}{|c|c|c|c|c|}
\hline \multicolumn{5}{|c|}{ Abstract IDDF2018-ABS-0086 Table 1} \\
\hline \multirow[t]{3}{*}{ Variables } & \multicolumn{4}{|c|}{ All the participants $(n=264)$} \\
\hline & \multicolumn{2}{|l|}{ Model 1} & \multicolumn{2}{|c|}{ Model 2} \\
\hline & HR $(95 \% \mathrm{Cl})$ & $p$ value & HR $(95 \% \mathrm{Cl})$ & $p$ value \\
\hline P/S ratio & $\begin{array}{c}0.000(0.000- \\
0.008)\end{array}$ & $<0.001$ & & \\
\hline $\begin{array}{l}\text { Pancreas } \\
\text { attenuation }\end{array}$ & & & $\begin{array}{c}0.865(0.816- \\
0.918)\end{array}$ & $<0.001$ \\
\hline Creatinine & $\begin{array}{c}1.003(1.000- \\
1.006)\end{array}$ & 0.033 & $\begin{array}{c}1.003(1.000- \\
1.006)\end{array}$ & 0.025 \\
\hline Calcium & $\begin{array}{c}0.920(0.165- \\
5.117)\end{array}$ & 0.924 & $\begin{array}{c}0.769(0.153- \\
3.853)\end{array}$ & 0.749 \\
\hline Albumin & $\begin{array}{c}0.963(0.887- \\
1.046)\end{array}$ & 0.375 & $\begin{array}{c}0.969(0.895- \\
1.049)\end{array}$ & 0.440 \\
\hline
\end{tabular}

Conclusions The pancreas attenuation level and the $\mathrm{P} / \mathrm{S}$ ratio are valuable predictors of the severity, mortality, and systemic complications in patients with AP.

\section{IDDF2018-ABS-0087 SERUM METABOLITE PROFILING OF FAMILIAL ADENOMATOUS POLYPOSIS USING UPLC-MS/MS}

${ }^{1}$ Sun Liyan*, ${ }^{2}$ Kang Qian, ${ }^{2}$ Jin Peng, ${ }^{2}$ Xu Junfeng, ${ }^{2}$ Pan Yuanming, ${ }^{2}$ Sheng Jianqiu. ${ }^{1}$ Chinese PLA General Hospital, China; ${ }^{2}$ PLA Army General Hospital, China

\subsection{6/gutjnl-2018-IDDFabstracts. 102}

Background Familial adenomatous polyposis (FAP) is an autosomal dominantly inherited intestinal polyposis syndrome accounts for about $1 \%$ of all colorectal cancers (CRC). Despite increasing awareness of its molecular pathogenesis? over the past two decades, there is still a lack of reliable biomarkers for the screening, monitoring and treatment of FAP? In this study, based on metabonomics analysis, we aimed to identify candidate biomarkers that can be used for FAP detection with high sensitivity and specificity by using human serum samples.

Methods Serum metabolites from FAP patients $(\mathrm{N}$ ? =25) and healthy subjects $(n=14)$ were profiled using Ultra Performance Liquid? Chromatography and Tandem Mass Spectrometry (UPLC-?MS/MS).

Results 125 metabolites (down-regulated 78 and up-regulated 47) were identified with statistical tests of? orthogonal partial least-squares-discriminant analysis (OPLS-DA), with the conditions of variable importance in projection (VIP) $>1, p<0.05$ using the Mann-? Whitney $U$ test, and fold change $(\mathrm{FC}) \geq 2$ or.

Abstract IDDF2018-ABS-0087 Table 1 The serum metabolites showing statistical significance

\begin{tabular}{lccc}
\hline Group name & $\begin{array}{c}\text { All significant } \\
\text { difference }\end{array}$ & $\begin{array}{c}\text { Down- } \\
\text { regulated }\end{array}$ & $\begin{array}{c}\text { Up- } \\
\text { regulated }\end{array}$ \\
\hline group1_vs_group2 & 125 & 78 & 47 \\
\hline
\end{tabular}

Conclusions We observed a distinct metabolic signature in FAP patients including tricarboxylic acid (TCA) cycle, amino acids metabolism, fatty acids metabolism, and bile acids (BAs) metabolism. Our results demonstrated that a panel of serum metabolite markers is of great value as a non-invasive diagnostic method for detecting FAP, and can be further used to study new therapeutic strategies.

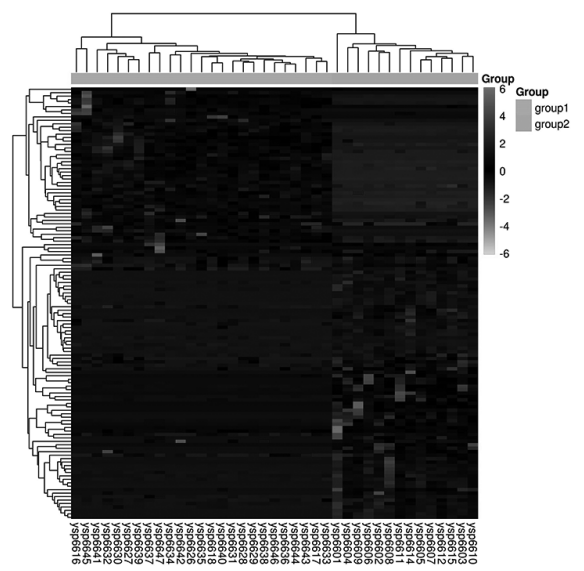

